\title{
Actitud del alumnado hacia la investigación en educación: Trabajando con vídeos en estudios de grado
}

\author{
Ma Jesús Gallego Arrufat $^{a}$ y Cristina Díaz-Martín ${ }^{b}$ \\ Rebut: 09/06/2015 Acceptat: 19/06/2015
}

\section{Resumen}

El trabajo presenta un estudio sobre la actitud del alumnado hacia la investigación en educación en estudios de grado. Para estudiar la actitud del alumnado se opta por la adaptación de un cuestionario que integra una escala de actitudes hacia la investigación (Papanastasiou, 2005). La adaptación es validada en el presente estudio por expertos en investigación educativa (escala Attitude Towards Research-Education, ATR-ED). Se realiza un diseño cuasiexperimental pretest-postest con una muestra de 26 estudiantes de grado de la Universidad de Grandada, España. El ATR-ED se aplica al inicio y al final del semestre en una asignatura de Tecnologías de la Información y Comunicación (TIC) de grado. El programa de la asignatura incluye el trabajo con artículos y vídeos de investigación científica con el fin de fomentar el conocimiento del alumnado hacia la investigación en educación y mejorar su actitud. El análisis y la comparativa de los datos obtenidos tras las aplicaciones del ATR-ED presentan una mejora de la actitud del alumnado. Igualmente se deduce que los recursos audiovisuales proporcionados en la asignatura, junto a la metodología em pleada, han provocado un efecto positivo en la actitud. En el futuro se necesita investigar sobre video y flipped classroom

Palabras claves: (de Thesauro UNESCO): Actitudes hacia la investigación, Investigación en educación, Investigación sobre medios, Estudiante universitario, Vídeo educativo

\footnotetext{
a Universidad de Granada

b Universidad de Granada
} 


\title{
Undergraduate university students' attitude towards research in Education. Working with audiovisual media
}

\begin{abstract}
This work presents a study of student attitudes towards educational research in studies of degree. Student attitudes towards educational research have great importance for the academic development and professional practice. To study the student attitudes, a scale ATR-ED is adapted and assessed by expert educational researchers. This scale is based on an earlier questionnaire (Papanastasiou, 2005). A quasi-experimental pretestpostest design involving 26 students from studies of degree of the University of Granada, Spain is developed. The ATR-ED is applied to the beginning and the end of a university course on Information and Communication Technology. The course syllabus is constituted by activities about research articles and videos in order to improve knowledge and student attitudes towards educational research. The analysis and comparison of both ATR-ED data yield an improvement of the student attitudes. Likewise, audiovisual resources involved in the course have caused a positive effect in the student attitudes, along with the methodology used, have had a positive effect on the student attitudes. Future studies on video and flipped classroom are need.
\end{abstract}

Keywords: (de ERIC) Attitude Towards Research, Educational Research, Media Research, Undergraduate Students, Video Technology.

\section{Introducción}

El estudio analiza las actitudes hacia la investigación de estudiantes de grado que trabajan en clase vídeos de investigación educativa. En la actualidad es un tema especialmente interesante en educación superior y en el marco de la sociedad del conocimiento.

El punto de partida reside en que las instituciones universitarias son el principal motor de producción científica. Los estudios científicos han evolucionado y su impacto en el desarrollo de la comunidad global es cada vez más notable. En este contexto, la sociedad del conocimiento considera el saber y la tecnología como los elementos de mayor impacto para el desarrollo económico y social (Mora, 2004).

Los cambios en las instituciones universitarias desde su integración en el Espacio Europeo de Educación Superior (EESS) obligan a una mayor adaptación a la sociedad digital en la que el uso de los media es de gran importancia. Junto a ello destaca la repercusión del valor social del conocimiento científico. La tecnología e Internet en particular son el motor de cambio en el mundo de la educación superior (Duart y Mengual-Andrés, 2014). 
Además la investigación juega un papel muy importante en la formación de los estudiantes universitarios (Bate, 2010). Por estas razones surge la necesidad de abordar el estudio de la actitud hacia la investigación de la población universitaria.

La investigación en educación puede ser entendida como un proceso de innovación y cambio (Gitlin et al, 1999). En la práctica educativa y el aprendizaje del alumnado la investigación en educación juega un papel clave (Joram, 2007). El presente estudio se basa en la idea de que la investigación en educación debe desempeñar un papel destacado en los estudiantes y en su futura experiencia.

La actitud del estudiante universitario hacia la investigación en educación es un tema relevante. Existe un planteamiento doble: por una parte la idea de que la investigación en educación es fundamental en el ámbito académico y profesional del estudiante y, por otra, que los estudiantes necesitan dar un sentido a lo que hacen. Kit Field (2011) apunta la importancia de la investigación en la práctica docente, es decir, los profesores necesitan de la investigación para dar sentido al trabajo realizado: "What works and why" (Field, 2011). La importancia de la investigación no sólo se refleja en el profesorado, también es importante para el estudiante universitario. Este motivo nos lleva a la pregunta: ¿Cómo es la actitud hacia la investigación en educación del alumnado universitario?

Algunos estudios han demostrado que el cambio de la actitud del alumno, ya sea hacia la investigación, hacia una asignatura o hacia el uso de nuevas tecnologías, es posible y puede mejorar de forma positiva si se trabaja para ello (Chen et al, 2012; Hussein \& Ara, 2013; Peng et al, 2006; Schare et al., 1991; Sever et al, 2013; Van der Linden, 2012). De ahí el estudio de las actitudes del alumnado hacia la investigación en educación. La revisión de artículos que tratan sobre la mejora de la actitud del alumnado muestra la necesidad de conocer la actitud de los estudiantes pregraduados hacia la investigación en educación y trabajar para conseguir un cambio positivo.

Los resultados de algunos estudios apuntan a que el profesor debe jugar un papel muy importante en la actitud investigadora de sus estudiantes (Ekiz, 2006) y muestran que la actitud del profesorado y alumnado hacia la investigación debe unir la práctica y la investigación (Ekiz, 2006; Joram, 2007). Se puede afirmar que la práctica docente y la investigación van unidas. Además, docentes y estudiantes en período de formación también deben ser investigadores.

La práctica y el estudio de la investigación en educación necesitan que los estudiantes estén comprometidos. La mejora de la actitud y el interés del estudiante es clave para conseguir su compromiso en la investigación (Cosgun, 2013). Esta mejora unida a programas dedicados a motivar al alumnado hacia la investigación han demostrado que existen cambios positivos y el alumnado mejora su visión hacia la investigación (Papanastasiou, 2005; Cosgun, 2013; Bibi, Muhammad \& Majid, 2013; Van der Linden, 2012). Por lo tanto, el conocimiento de la investigación puede mejorar la actitud de los futuros graduados hacia la investigación en educación. También la práctica de la investigación puede llevar al alumnado a mejorar su actitud y sus habilidades (Van der Linden, 2015). 
La cuestión está en cómo mejorar la actitud de los estudiantes y qué programa, curso o herramientas se pueden utilizar para ello. En base a los argumentos dados, se opta por elegir una herramienta que permita al alumnado conocer la investigación en educación a través de recursos que se muestran en Internet. Una de las herramientas que puede conseguir despertar el interés del alumnado por la investigación y el método científico es el vídeo (Bartolomé, 2008; Cebrián y Gallego, 2011).

El vídeo es un recurso popular para los estudiantes de educación superior y sobre todo en la práctica educativa (So, Hung \& Yip, 2008). El vídeo es una herramienta que ofrece múltiples posibilidades para la enseñanza y la práctica en la investigación. Además, es uno de los soportes con mayor difusión de información (Bartolomé, 2008). La herramienta vídeo se elige en este trabajo gracias al material que se encuentra en este formato, si bien pensamos que en la actualidad por lo general las universidades no cuentan con suficientes recursos en vídeo sobre investigaciones en educación y la producción es incipiente (ejs. UGRmedia, Portal de Recursos Multimedia de la Universidad de Granada; Canal UGR http:/// ${ }^{\mathrm{a}}$

El vídeo no sólo es una herramienta de difusión sino que es soporte para el aprendizaje de los estudiantes y éstos lo valoran positivamente (Sever et al, 2013). También puede ayudar a captar el interés del alumno hacia la investigación en educación (Chen \& Cowie, 2014). Sin duda, los vídeos son más atractivos que los materiales digitalizados o textos (García-Fernández, 2011, 69). Por esta razón, en el estudio se trabaja con vídeos de investigación para mejorar la actitud del alumnado. Además, numerosos autores afirman que esta herramienta sirve de apoyo al aprendizaje y ayuda a captar el interés sobre cualquier tema que se trabaje (Bartolomé, 2008; So, Hung \& Yip, 2008; García-Fernández, 2011; Sever et al, 2013; Blomberg et al, 2014; Chen \& Cowie, 2014).

Es frecuente que el alumnado de grado desconozca qué se está investigando en su facultad. Los estudiantes están más preocupados por memorizar y reproducir contenidos para los exámenes (Hussein \& Ara, 2013) que por investigar para producir una mejora. Para estudiar el problema se adapta un cuestionario ATR-ED validado mediante expertos. La hipótesis de partida es que los estudiantes tienen bajo interés por la investigación educativa y que los materiales audiovisuales y los canales multimedia universitarios pueden ser un excelente vehículo de propagación de información sobre investigación en educación y ayudar al alumno a mejorar su actitud. El procedimiento incluye la elaboración de una guía previa de programación de la asignatura, que presenta artículos y vídeos de investigación en educación y que el alumnado trabaja en ella.

El objetivo fundamental es fomentar la visibilidad de la investigación en educación a través de recursos audiovisuales. Los objetivos específicos son:

- Conocer y analizar la actitud de los estudiantes de grado en Ciencias de la Educación en investigación educativa.

- Fomentar el conocimiento de la investigación a través de vídeos educativos. 


\section{Método}

El trabajo presenta un análisis del interés del alumnado hacia la investigación en educación mediante un diseño cuasi-experimental pretest-postest (Campbell \& Stanley, 1973; Ato \& Vallejo, 2015) con un grupo de estudiantes de grado.

Para este estudio se adapta una escala denominada "Actitudes hacia la investigación en educación" ATR-ED, tomando como base los cuestionarios ATR de Papanastasiou, 2005; Cosgun, 2013; Hussein \& Ara, 2013. Otros ítems del ATR-ED se elaboran a partir de los estudios de Boscolo (2009) e Ibáñez (2013).

\section{Participantes}

Los participantes son 26 estudiantes de 20-31 años de la Universidad de Granada, seleccionados mediante un muestreo intencional (Bisquerra, 2004). Cursan una asignatura de Tecnologías de la Información y Comunicación (TIC) en el grado.

\section{Procedimiento}

El estudio se realiza en las fases que se muestran en la Figura 1.

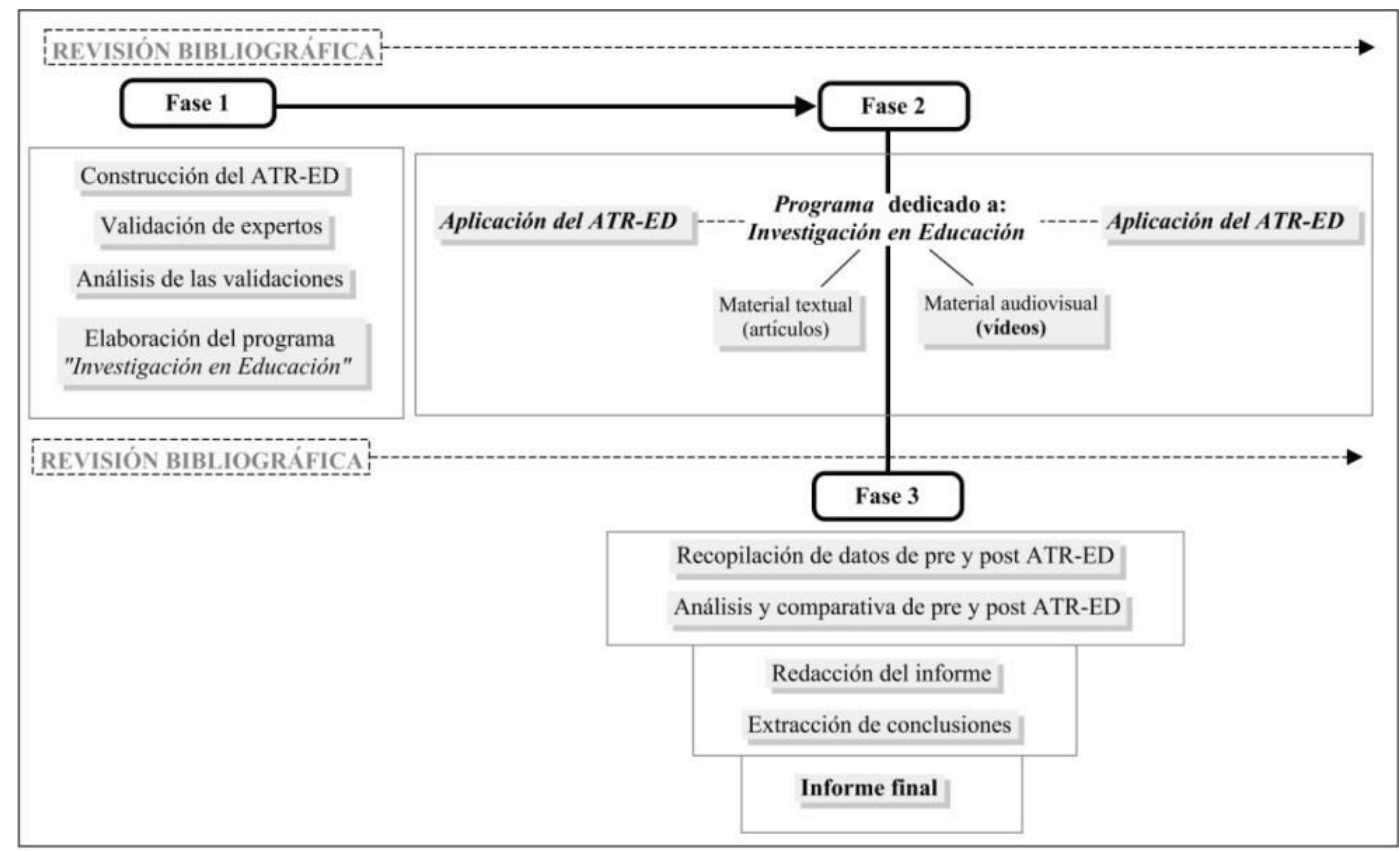

Figura 1. Fases del estudio de investigación

\section{Adaptación y validación del cuestionario}

El proceso de validación del cuestionario se lleva a cabo en el primer mes de clases, conforme a la siguiente secuencia:

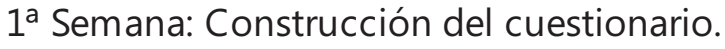


UT. Revista de Ciències de l'Educació

2015 num. 1. Pag. 8-29
Actitud del alumnado hacia la investigación en educación: Trabajando con vídeos en estudios de grado

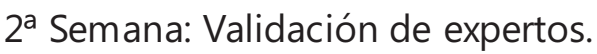

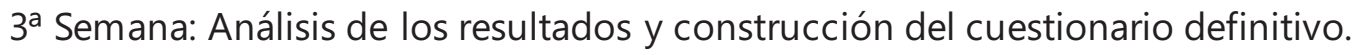

Teniendo en cuenta el problema de investigación y la bibliografía revisada se opta por construir una ATR, escala de actitudes hacia la investigación o "Attitude Towards Research" (Cosgun, 2013; Hussein y Ara, 2013; Papanastasiou, 2005). En total se presentan 60 ítems distribuidos en siete dimensiones a valorar según los criterios de univocidad, pertinencia y grado de importancia (escala de 1 a 5). Se añade un apartado de observaciones en cada ítem para su eliminación o reescritura.

Validación del cuestionario "Actitudes hacia la Investigación en Educación" (ATR-ED)

Se envía a expertos en educación y en particular en tecnología educativa y metodología de la investigación. El análisis de las validaciones, nueve en total, se realiza la $3^{a}$ semana. Según la validación, los ítem s cumplen los criterios de univocidad y pertinencia y el grado de valoración $\leq 3$. En concreto se eliminan cuatro ítems y se reescriben 15 siguiendo las recomendaciones de los expertos. Todas las dimensiones son valoradas positivamente, excepto una única vez "Ansiedad Investigadora" (ninguna de las restantes mencionan este apartado).

Tras el análisis de las nueve validaciones se construyó el cuestionario ATR-ED definitivo, compuesto de 56 ítems distribuidos en siete dimensiones:

- Utilidad de la investigación: beneficios de la investigación para el desarrollo académico, profesional y personal del estudiante.

- Ansiedad por investigar: preocupación del estudiante por la investigación.

- Actitudes positivas hacia la investigación: intereses de los estudiantes hacia la investigación.

- Relevancia de la investigación en la vida: importancia de la investigación para la vida del estudiante.

- Formación en investigación: importancia y necesidad de la formación en investigación de los estudiantes y futuros profesionales.

- Motivación hacia la investigación: incentivación del estudiante hacia la investigación.

- Medios de investigación: uso de fuentes de información y recursos audiovisuales de investigación.

En la Tabla 1 se muestran los resultados de la validación en cada una de las dimensiones: 


\begin{tabular}{lccc}
\hline Dimensión & Items N & Modificación \% & Supresión \% \\
\hline Utilidad de la investigación (UI) & 15 & 20 & - \\
Ansiedad por investigar (AI) & 6 & 16 & 33,4 \\
Actitudes positivas hacia la investigación (AP) & 8 & 37,5 & - \\
Relevancia dela investigación en la vida (RV) & 5 & 80 & - \\
Formación en investigación (FI) & 9 & 55,5 & - \\
Motivación hacia la investigación (MI) & 8 & 12,5 & 11,5 \\
Medios de investigación (MMI) & 9 & 33,3 & 11,2 \\
\hline
\end{tabular}

Tabla 1. Resultados de la validación del ATR-ED

En general, la modificación de los ítems del cuestionario ha supuesto un $25 \%$ mientras que el $75 \%$ restante no ha sido modificado. Solamente se suprime $4 \%$ de ítems, por lo que el cuestionario adaptado y aplicado contiene el $96 \%$ de los ítems de la escala original.

\section{Administración del cuestionario ATR-ED}

El cuestionario adaptado y validado se administra al alumnado una vez iniciada la asignatura y tras finalizar el período de alteración de matrícula, para conseguir que los mismos estudiantes realicen el cuestionario al inicio y al final del semestre.

En la asignatura se elabora un programa para trabajar vídeos y artículos de investigación en educación, seleccionando artículos en diferentes revistas indexadas y vídeos de investigación en "Youtube". En algún caso, vídeo y artículo de la misma revista, ej. "Education Policy Analysis Archives" (http://epaa.asu.edu/ojs/). El programa se sigue durante 10 semanas, tras las cuales se vuelve a administrar el cuestionario. El postest se realiza al finalizar el semestre usando formulario de Google. Los resultados son analizados empleando Microsoft Office Excel.

\section{Resultados}

Los datos recogidos en el pretest y postest (ATR-ED anterior y ATR-ED posterior) muestran resultados cuantitativos sobre la actitud de los estudiantes hacia la investigación en educación. Los resultados aparecen en las tablas 2 y 3 , según una escala de valoración tipo Likert de 5 niveles: totalmente en desacuerdo, en desacuerdo, ni de acuerdo ni en desacuerdo, de acuerdo y totalmente de acuerdo. Los datos de am bos ATR-ED (pre- y post-) son comparados mediante el cálculo del porcentaje de alumnado que se encuentra en los distintos niveles de valoración. La Tabla 2 presenta los resultados de la aplicación previa del ATR-ED. 


\begin{tabular}{|c|c|c|c|c|c|}
\hline & $\begin{array}{l}\text { Totalmente } \\
\text { en } \\
\text { desacuerdo }\end{array}$ & $\begin{array}{l}\text { En } \\
\text { desacuerdo }\end{array}$ & $\begin{array}{l}\mathrm{Ni} \text { de } \\
\text { acuerdo ni en } \\
\text { desacuerdo }\end{array}$ & $\begin{array}{l}\text { De } \\
\text { acuerdo }\end{array}$ & $\begin{array}{l}\text { Totalmente } \\
\text { de acuerdo }\end{array}$ \\
\hline Utilidad de la investigación & 0.0 & 2.6 & 15.1 & 48.5 & 33.8 \\
\hline Ansiedad investigadora & 1.9 & 12.5 & 36.5 & 34.6 & 14.4 \\
\hline $\begin{array}{l}\text { Actitudes positivas hacia la } \\
\text { investigación }\end{array}$ & 4.3 & 16.8 & 35.6 & 33.2 & 10.1 \\
\hline $\begin{array}{l}\text { Relevancia de la } \\
\text { investigación en la vida }\end{array}$ & 0.8 & 6.9 & 37.7 & 34.6 & 20.0 \\
\hline Formación en investigación & 0.4 & 8.5 & 36.3 & 46.6 & 8.1 \\
\hline $\begin{array}{l}\text { Motivación hacia la } \\
\text { investigación }\end{array}$ & 0.0 & 4.9 & 39.0 & 45.6 & 10.4 \\
\hline Medios de investigación & 1.0 & 10.6 & 39.4 & 34.1 & 14.9 \\
\hline
\end{tabular}

Tabla 2. Resultados de la aplicación previa del ATR-ED

La Tabla 2 muestra la valoración de los estudiantes en porcentajes, según las dimensiones del cuestionario. El nivel más valorado (48.5\%) está en la dimensión "Utilidad de la investigación". En la dimensión "Ansiedad investigadora" el mayor porcentaje del alumnado no está de acuerdo ni en desacuerdo (36,5\%) y el menor porcentaje (1,9\%) está totalmente en desacuerdo. Por otro lado, el 35,6\% del alumnado se sitúa en el valor 3 (ni de acuerdo ni en desacuerdo) en "Actitudes positivas hacia la investigación" y el menor porcentaje está totalmente en desacuerdo (4,3\%). En la dimensión "Relevancia de la investigación en la vida" 37,7\% no está ni de acuerdo ni en desacuerdo. En las dimensiones "Formación en investigación" y "Motivación hacia la investigación" más del $45 \%$ del alumnado se encuentra en el nivel de acuerdo. La última dimensión reflejada en la Tabla 2 presenta un 39,4\% del alumnado ni de acuerdo ni en desacuerdo. Las tres últimas dimensiones siguen reflejando el mínimo porcentaje de alumnado totalmente en desacuerdo.

La Tabla 3 presenta los resultados de la aplicación posterior del ATR-ED.

\begin{tabular}{|c|c|c|c|c|c|}
\hline & $\begin{array}{l}\text { Totalmente } \\
\text { en } \\
\text { desacuerdo }\end{array}$ & $\begin{array}{l}\text { En } \\
\text { desacuerdo }\end{array}$ & $\begin{array}{l}\mathrm{Ni} \text { de } \\
\text { acuerdo ni en } \\
\text { desacuerdo }\end{array}$ & $\begin{array}{l}\text { De } \\
\text { acuerdo }\end{array}$ & $\begin{array}{l}\text { Totalmente } \\
\text { de acuerdo }\end{array}$ \\
\hline Utilidad de la investigación & 0.5 & 1.3 & 17.4 & 42.1 & 38.7 \\
\hline Ansiedad investigadora & 1.9 & 13.5 & 34.6 & 26.0 & 24.0 \\
\hline $\begin{array}{l}\text { Actitudes positivas hacia la } \\
\text { investigación }\end{array}$ & 7.2 & 13.5 & 36.5 & 28.4 & 14.4 \\
\hline $\begin{array}{l}\text { Relevancia de la } \\
\text { investigación en la vida }\end{array}$ & 1.5 & 6.9 & 34.6 & 31.5 & 25.4 \\
\hline Formación en investigación & 2.1 & 6.0 & 35.9 & 41.0 & 15.0 \\
\hline $\begin{array}{l}\text { Motivación hacia la } \\
\text { investigación }\end{array}$ & 1.6 & 4.9 & 33.0 & 44.0 & 16.5 \\
\hline Medios de investigación & 3.4 & 7.7 & 31.3 & 37.5 & 20.2 \\
\hline
\end{tabular}

Tabla 3. Resultados de la aplicación posterior del ATR-ED

En esta ocasión, la dimensión "Motivación hacia la investigación" presenta el mayor porcentaje de alumnado que está de acuerdo (44\%). "Ansiedad investigadora", 
"Actitudes positivas hacia la investigación" y "Relevancia de la investigación en la vida" muestran entre 36,5\% y 34,6\% de alumnado ni de acuerdo ni en desacuerdo. "Formación en investigación" presenta $41 \%$ de alumnado de acuerdo. Por último, la dimensión "Medios de investigación" representa el mayor porcentaje de alumnado de acuerdo (37,5\%). El nivel totalmente en desacuerdo muestra el menor porcentaje en todas las dimensiones.

En el resultado del postest la dimensión mejor valorada es la "Utilidad de la investigación", ya que el $80,8 \%$ de los estudiantes está de acuerdo o totalmente en acuerdo. Era la más valorada también en el ATR-ED anterior. En la dimensión "Actitudes positivas hacia la investigación" aumenta tanto el porcentaje de estudiantes de acuerdo $(33,2$ frente a 28,4$)$ como en desacuerdo $(16,8$ frente a 15,5$)$.

A continuación se muestran las diferencias entre ambos cuestionarios ATR-ED, según las dimensiones del cuestionario:

a) Utilidad de la investigación.

La Figura 2 muestra la comparativa entre ambos ATR-ED en la dimensión "Utilidad de la investigación".

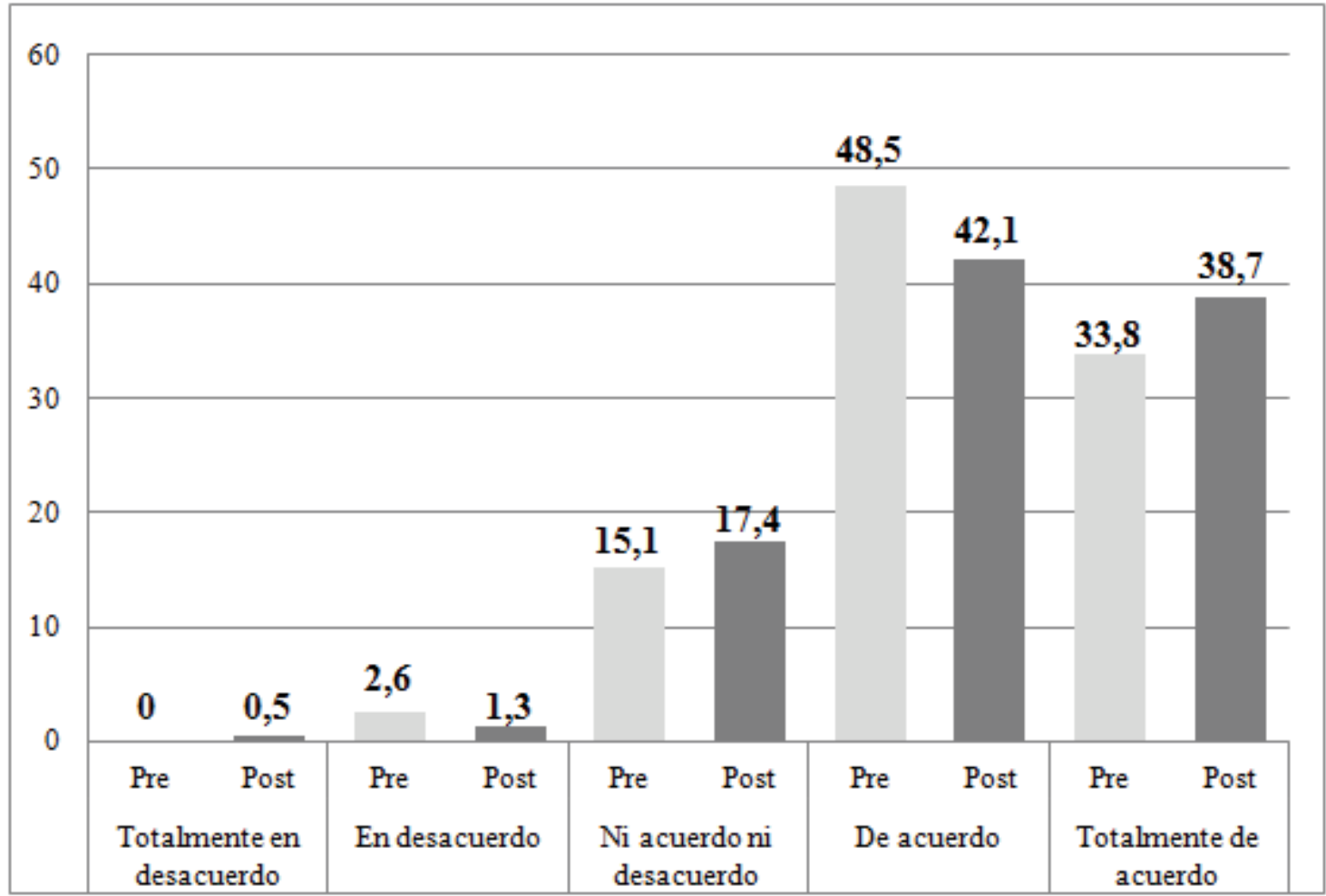

Figura 2. Comparativa entre ambos ATR-ED en la dimensión "Utilidad de la Investigación" 
El porcentaje es más alto en el previo que en el posterior en los niveles en desacuerdo $y$ de acuerdo. Los niveles que presentan mayor diferencia en los porcentajes son de acuerdo y totalmente de acuerdo. El previo muestra 48,5\% de alumnado de acuerdo, frente a $42,1 \%$ en el posterior. Se eleva también el porcentaje de totalmente de acuerdo (33,8\% frente a $38,7 \%)$.

b) Ansiedad investigadora

La Figura 3 muestra la comparativa entre ambos ATR-ED en la dimensión "Ansiedad investigadora".

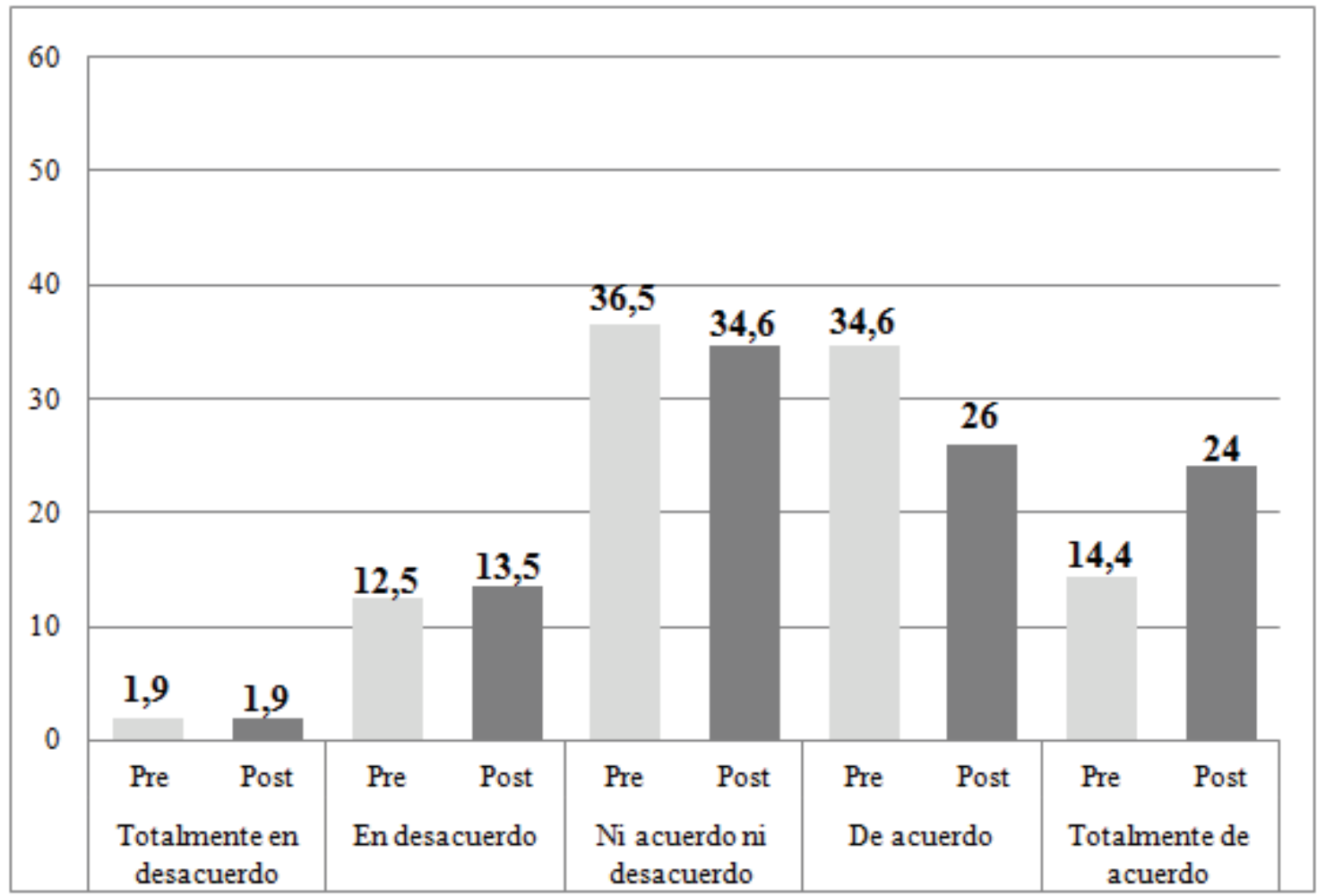

Figura 3. Comparativa entre ambos ATR-ED en la dimensión "Ansiedad investigadora"

Aquí aumentan los niveles en desacuerdo y totalmente de acuerdo. Los niveles que presentan mayor diferencia en los porcentajes son de acuerdo (34,6\% frente a $26 \%$ ), que disminuye considerablemente mientras que totalmente de acuerdo aumenta (14,4\% frente a $24 \%$ de alumnado). 
c) Actitudes positivas hacia la investigación

La Figura 4 muestra la comparativa en la dimensión "Actitudes positivas hacia la investigación".

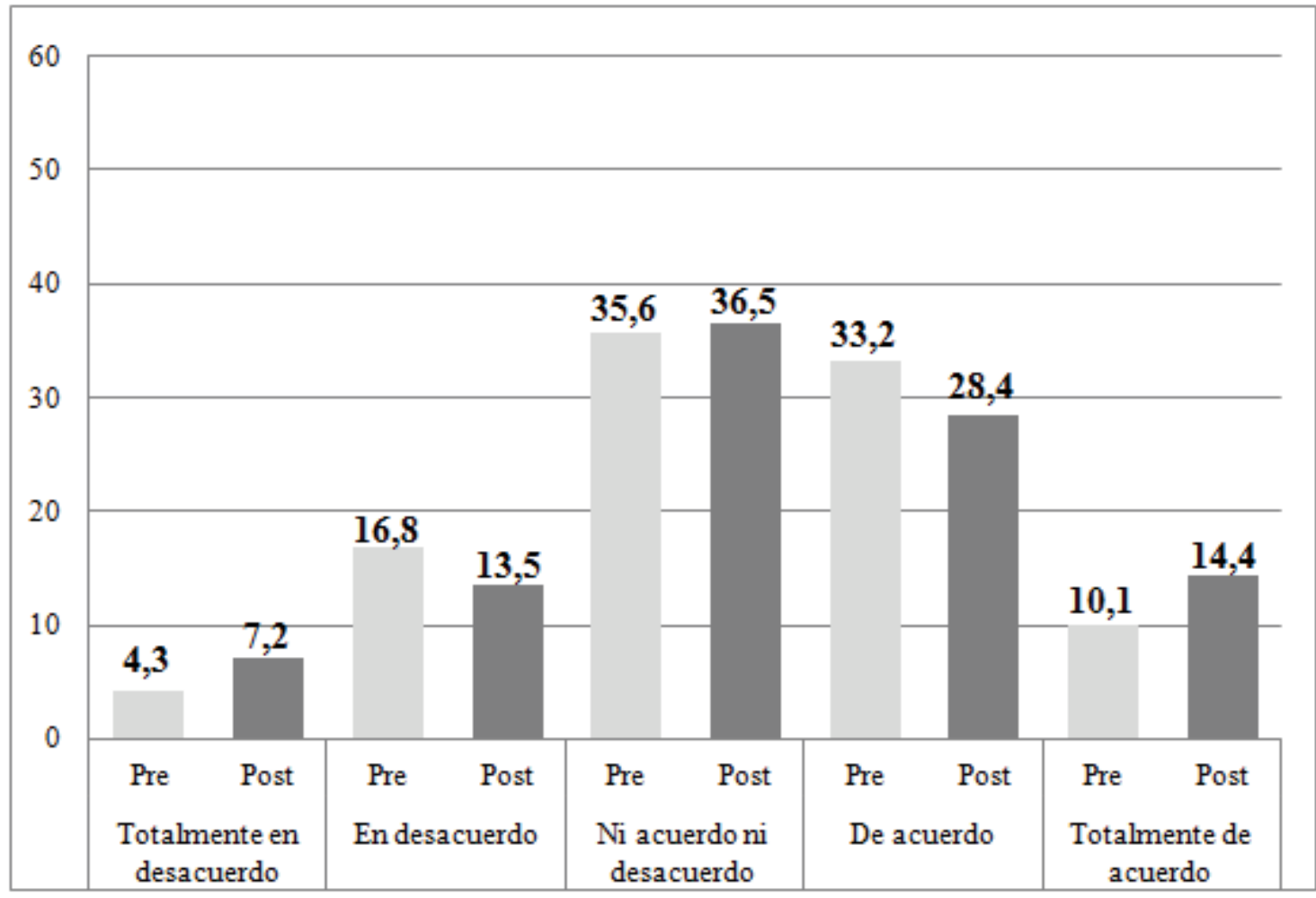

Figura 4. Comparativa entre ambos ATR-ED en la dimensión "Actitudes positivas hacia la investigación"

Como se aprecia en la Figura 4 aumentan los niveles más extremos de la escala Likert (1) y (5) mientras que casi se mantiene el nivel central (35,5\% frente a $36,5 \%)$. Se observa que en el ATR-ED posterior el porcentaje en los niveles totalmente en desacuerdo y totalmente de acuerdo es más elevado. Por el contrario, el porcentaje de alumnado en los niveles en desacuerdo y de acuerdo disminuye en el ATR-ED posterior. El nivel de acuerdo disminuye 4,8 (33,2\% frente a 28,4\%) y el nivel totalmente de acuerdo aumenta en proporción similar $(4,3)$ en los ATR-ED previo-posterior $(10,1 \%$ frente a $14,4 \%)$. 
d) Relevancia de la investigación en la vida

La Figura 5 presenta la comparativa en la dimensión "Relevancia de la investigación en la vida".

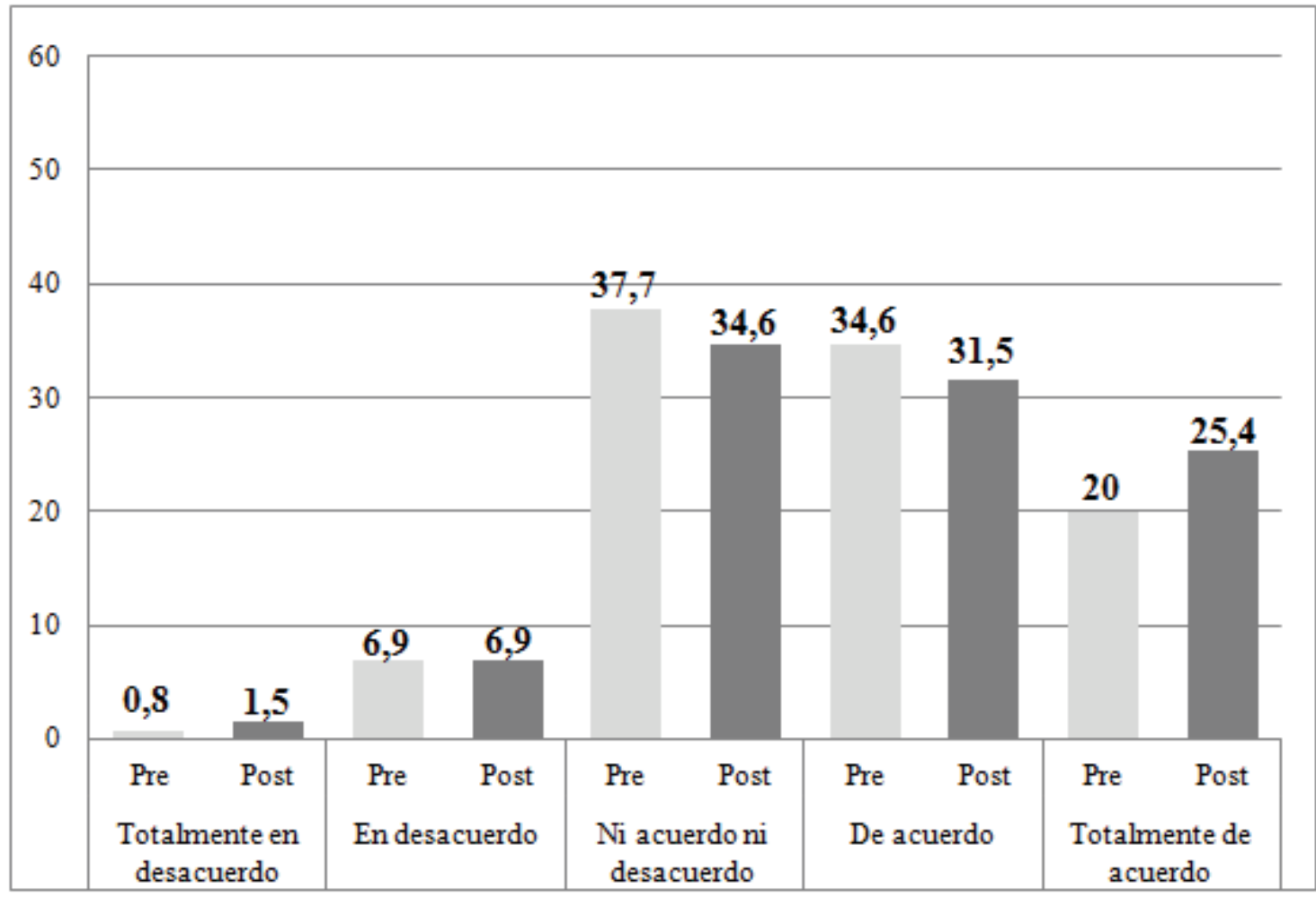

Figura 5. Comparativa entre ambos ATR-ED en la dimensión "Relevancia de la investigación en la vida"

En esta dimensión la variación observada muestra una tendencia interesante, si bien los niveles ni acuerdo ni desacuerdo y de acuerdo muestran un porcentaje menor en el ATR-ED posterior. El ATR-ED posterior presenta un porcentaje de alumnado mayor en los niveles totalmente en desacuerdo y totalmente de acuerdo (también los extremos, como en la dimensión "Actitudes positivas hacia la investigación"). Este último nivel presenta mayor variación en el porcentaje (20\% frente a $25,4 \%$ ), lo que significa una diferencia de 5,4 entre ambos ATR-ED. 
e) Formación en investigación

La Figura 6 presenta la comparativa entre ambos ATR-ED en la dimensión "Formación en investigación".

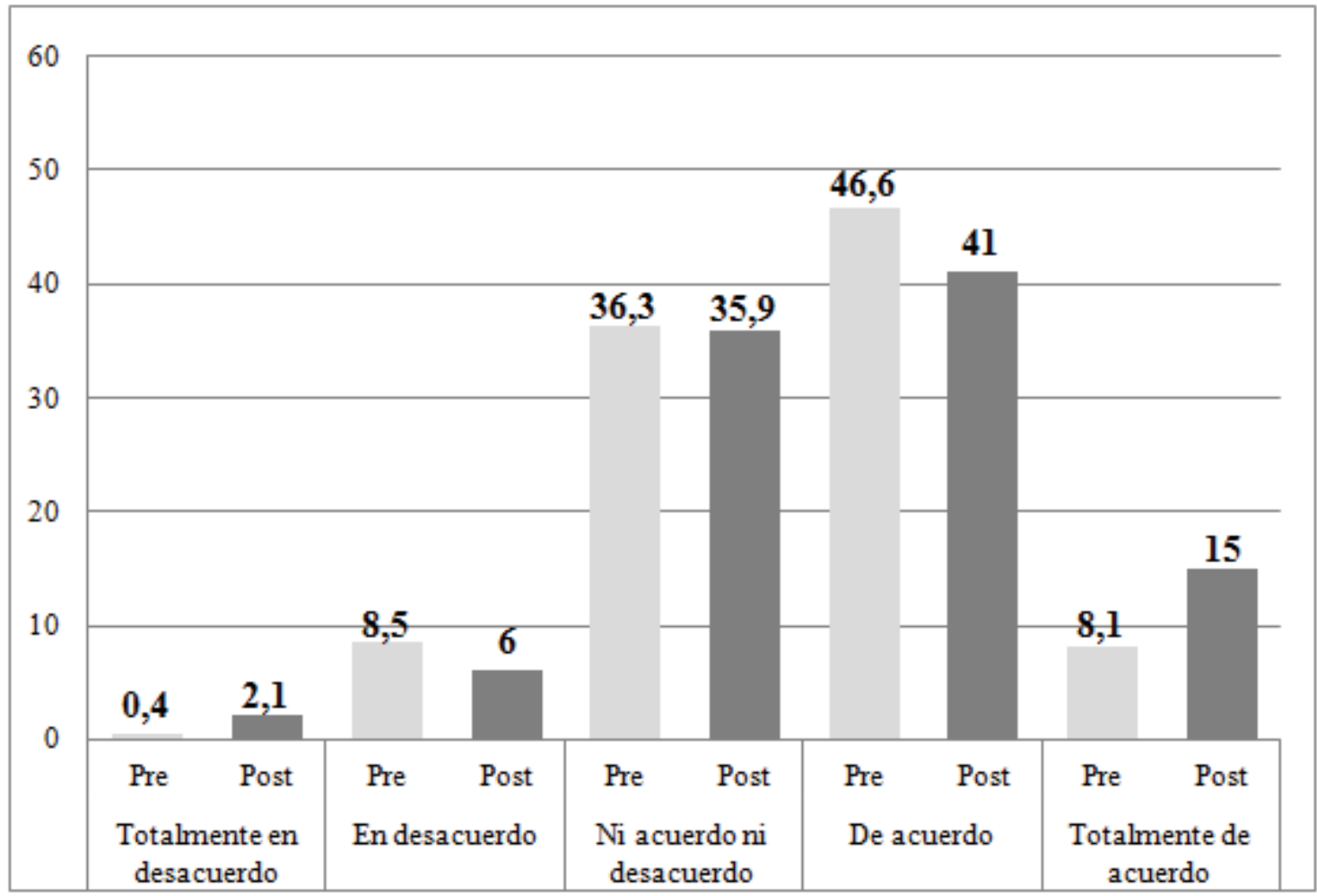

Figura 6. Comparativa entre ambos ATR-ED en la dimensión "Formación en investigación"

En la Figura 6 se aprecia una tendencia similar: aumentan los niveles más extremos de la escala tipo Likert, disminuyen los niveles en desacuerdo y de acuerdo, mientras que permanece inalterable el nivel ni acuerdo ni desacuerdo. El ATR-ED posterior muestra menor porcentaje de alumnado en los niveles en desacuerdo ( $8,5 \%$ frente a $6 \%$ ) y de acuerdo (46,6\% frente a $41 \%)$. Al contrario, presentan un aumento totalmente en desacuerdo y totalmente de acuerdo, apareciendo en éste último nivel la variación más elevada $(6,9)$. Esta diferencia supone, junto con la obtenida en el nivel de acuerdo $(5,6)$ la mayor variación, aumentando el nivel de totalmente de acuerdo al tiempo que disminuye el de acuerdo. 
f) Motivación hacia la investigación

La Figura 7 presenta la comparativa entre ambos ATR-ED en la dimensión "Motivación hacia la investigación".

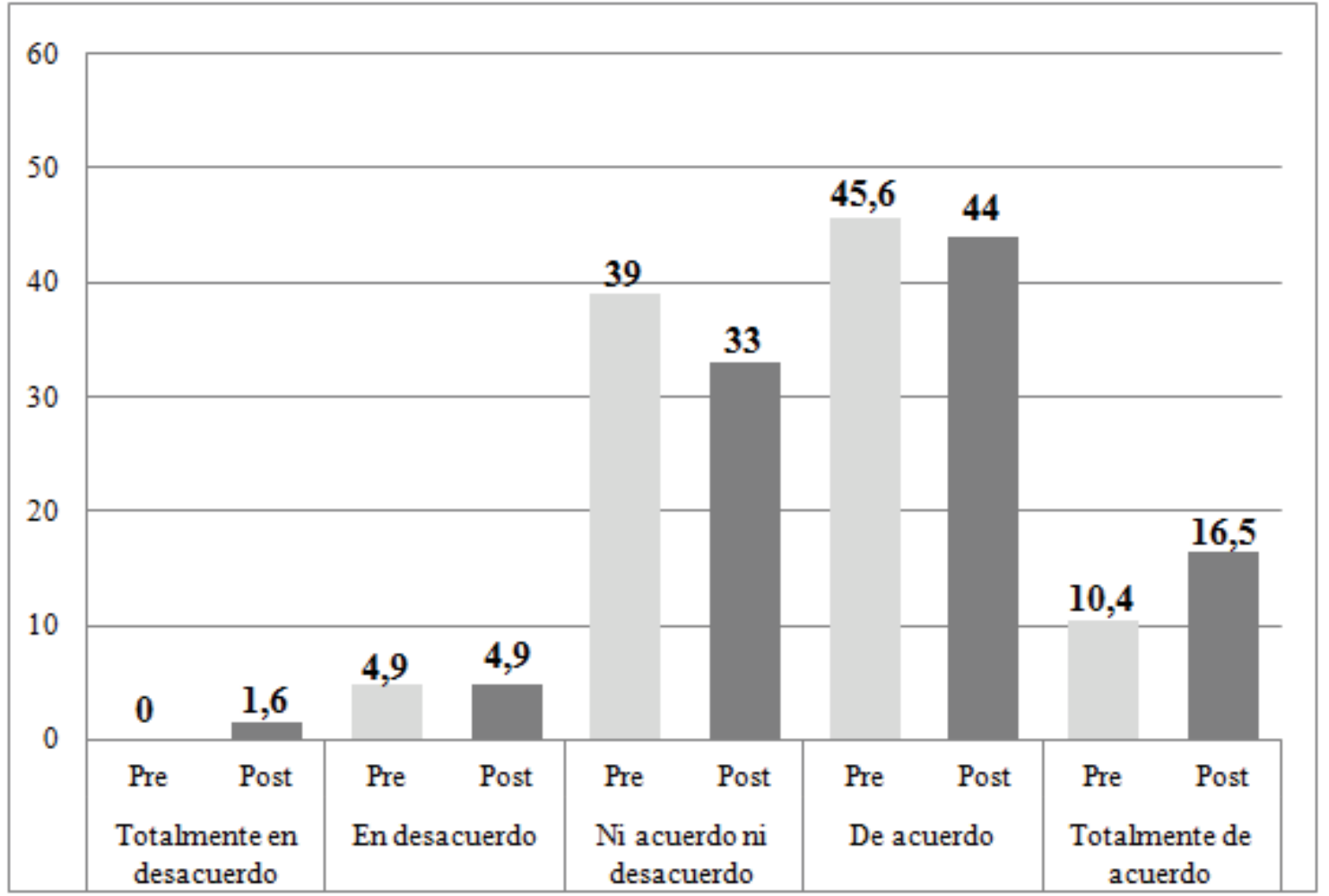

Figura 7. Comparativa entre ambos ATR-ED en la dimensión "Motivación hacia la investigación"

En esta dimensión, al igual que en las anteriores, de nuevo el ATR-ED posterior muestra un porcentaje mayor de alum nado en los niveles totalmente en desacuerdo y totalmente de acuerdo con respecto al ATR-ED previo. En concreto, los niveles que presentan mayor diferencia en los porcentajes son ni acuerdo ni desacuerdo, que disminuye (39\% frente a $33 \%)$, y totalmente de acuerdo, que aumenta (10,4\% frente a $16,5 \%)$. 
g) Medios de investigación

La Figura 8 presenta la comparativa entre ambos ATR-ED en la dimensión "Medios de investigación".

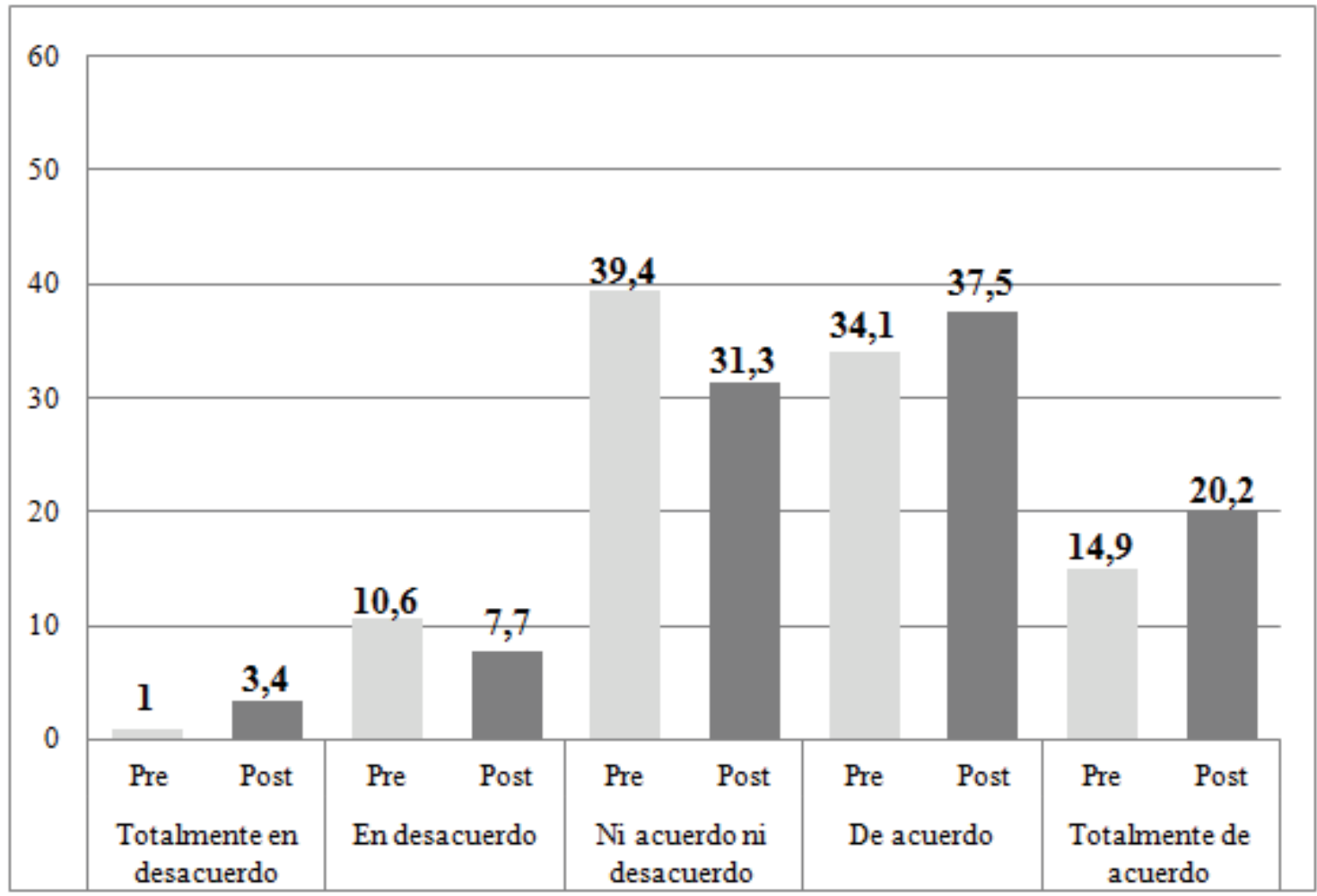

Figura 8. Comparativa entre ambos ATR-ED en la dimensión "Medios de investigación"

En esta dimensión el porcentaje de alumnado en los niveles en desacuerdoy niacuerdo nidesacuerdo disminuye en el ATR-ED posterior, mientras que el resto aumentan. Existe mayor diferencia $(8,1)$ en el nivel central ni acuerdo ni desacuerdo, que disminuye $(39,4 \%$ frente a $31,3 \%)$ y en el nivel totalmente de acuerdo, que aumenta 5,3 (14,9\% frente a 20,2\%) en el ATR-ED posterior.

\section{Discusión}

Según los resultados obtenidos en el primer ATR-ED aplicado se puede afirmar que la dimensión "Actitudes positivas hacia la investigación" es dispersa aunque se concentra ligeramente en los niveles 3 y 4 de la escala Likert, lo que está en línea con las observaciones de Hussein \& Ara (2013). El hecho de que la mayor parte del alumnado se encuentre en los niveles ni de acuerdo ni en desacuerdo y, en menor medida, de acuerdo en cuatro de las siete dimensiones podría caracterizarse como dudas o falta de perspectiva para emitir un juicio, si bien deberían realizarse entrevistas o grupos de 
discusión con los estudiantes para afirmar esto. En las tres dimensiones restantes (utilidad, formación y motivación) los estudiantes están en su mayoría de acuerdo en el ATR-ED previo, y sobre todo en la dimensión valorada de forma más positiva, "Utilidad de la investigación" (48,5\% de acuerdo). El alumnado de grado considera que la investigación en educación es útil.

En general, los resultados indican en el pretest de este estudio 56,7\% de estudiantes en los valores 1-2-3 de la escala tipo Likert. No puede afirmarse que el alumnado muestre una actitud positiva hacia la investigación en educación, por lo que se decide la elaboración de un programa que dedique parte de los contenidos y actividades a trabajar el tema con artículos y vídeos de investigación en educación, en la medida en que se puede ayudar a los estudiantes a mejorar la actitud (Papanastasiou, 2005; Cosgun, 2013).

Los datos resultantes de la aplicación del ATR-ED posterior presentan interesantes diferencias respecto al ATR-ED previo (Figuras 2-8). La dimensión mejor valorada sigue siendo "Utilidad de la investigación", aunque aumenta el porcentaje de alumnado en el nivel totalmente en desacuerdo. Por el contrario, cinco dimensiones ("Ansiedad investigadora", "Relevancia en la vida", "Formación en investigación", "Motivación hacia la investigación" y "Medios de investigación") aumentan el porcentaje de alumnado en los niveles de acuerdo y totalmente de acuerdo en el ATR-ED posterior. Gracias al programa seguido en clase y al papel del profesorado para la mejora de la actitud del alumnado hacia la investigación en investigación, los resultados mostrados en el ATRED posterior han sido más elevados en los niveles de acuerdoy totalmente de acuerdo. Se puede afirmar que la actitud del alumnado ha mejorado gracias al programa dedicado a la investigación en educación (Chen et al, 2012; Hussein \& Ara, 2013; Peng et al, 2006; Sever et al, 2013; Schare et al, 1991).

Un examen de cada una de las dimensiones y los ítems que las integran permite afirmar que en el postest la dimensión "Utilidad de la investigación" aumenta en porcentaje de alumnado en el nivel totalmente en desacuerdo, especialmente el ítem "La investigación está conectada con mis intereses en estos estudios". En la dimensión "Actitudes positivas hacia la investigación" aumenta el porcentaje de alumnado en el nivel totalmente en desacuerdo en los ítems "Me encanta la investigación", "Me interesa estudiar la investigación en profundidad", "La investigación es interesante", y "Estoy interesado en participar en proyectos de investigación". Los datos suponen que menos del $10 \%$ del alumnado ha bajado su interés hacia la investigación. El ítem "la investigación es relevante en mi vida" muestra un porcentaje de alumnado mayor de acuerdo en el ATRED posterior. En la dimensión "Formación en investigación" aumenta también el porcentaje de alumnado en el nivel totalmente en desacuerdo, lo cual puede interpretarse como una demanda; según el ítem "Los estudiantes de Ciencias de la Educación cuentan con suficiente formación en investigación" el mayor porcentaje de alumnado cree que la formación en investigación no es suficiente. También aumenta el porcentaje de alumnado totalmente en desacuerdo en la dimensión "Motivación hacia la investigación". En este caso, los ítems "la investigación incrementa la motivación de los estudiantes"y "las actividades desarrolladas en las asignaturas 
contribuyen a mi interés por la investigación educativa" supone un aumento del porcentaje de alumnado que no está motivado hacia la investigación en educación.

A pesar de que hay mayor porcentaje de alumnado con bajo interés y motivación hacia la investigación, el nivel totalmente de acuerdo aumenta su porcentaje. El ATR-ED previo muestra porcentajes más bajos en el nivel totalmente en desacuerdo. La dimensión "Utilidad de la investigación" presenta un porcentaje mayor en el nivel antes mencionado. Los ítems con mayor porcentaje de alumnado en este nivel son: " $L a$ investigación es útil para todos los profesionales", "Mientras investigo puedo desarrollar mi conocimiento", "la investigación debe desarrollar habilidades para resolver problemas". Los resultados del ATR-ED posterior muestran que el alumnado piensa que la investigación es útil y ayuda a resolver problemas y desarrollar su conocimiento. La Figura 3 muestra que el grado de totalmente de acuerdo en la "Ansiedad investigadora" aumenta significativamente en el ATR-ED posterior (14,4\% frente a $24 \%$ ). Según los ítems de esta dimensión, hay mayor porcentaje de alum nado con una actitud negativa ante la investigación porque aumenta su preocupación ante el tema, se sienten más inseguros y piensan que es un tema complejo. La dimensión "Actitudes positivas hacia la investigación" muestra un mayor porcentaje de alumnado que está interesado en la investigación y en leer estudios en su campo. "Formación en la investigación" presenta un porcentaje de alumnado mayor en los ítems "La formación en investigación educativa es una necesidad de los estudiantes universitarios para mejorar su aprendizaje" y "Los estudiantes o futuros profesionales de la educación deberían tener una formación amplia en investigación educativa". El alumnado piensa que es necesaria la formación en investigación. La dimensión "Motivación hacia la investigación" muestra un mayor porcentaje de alumnado en los ítems "La investigación incrementa la motivación de los estudiantes" $y$ "El profesorado propone diferentes temas para investigar en la realización de trabajos/proyectos científicos de clase". En el postest aparece un porcentaje más elevado de alumnado que está más motivado por investigar.

Un examen adicional de las actitudes del alumnado hacia la investigación en educación trabajando con vídeos, de acuerdo con la última dimensión "Medios de investigación", es realizado por dos razones: porque es la dimensión que puede llegar a mostrar si verdaderamente los vídeos de investigación han sido útiles, y porque es la dimensión que mayor diferencia presenta en el porcentaje de alumnado en los niveles de acuerdo y totalmente de acuerdo. La Tabla 3 muestra la comparativa de ambos ATR-ED en los niveles de acuerdo y totalmente de acuerdo obtenidos en la dimensión "Medios de investigación".

\begin{tabular}{lcc}
\hline & PRE ATR-ED & POST ATR-ED \\
\hline $\begin{array}{l}\text { Las fuentes de información sobre investigación en educación son útiles } \\
\text { Prefiero ver un vídeo que leer un artículo sobre investigación en }\end{array}$ & 76.9 & 76.9 \\
educación & $\mathbf{5 7 . 7}$ & $\mathbf{8 0 . 8}$ \\
$\begin{array}{l}\text { Los recursos audiovisuales fomentan el interés por la investigación } \\
\text { educativa }\end{array}$ & 69.2 & $\mathbf{8 8 . 5}$ \\
$\begin{array}{l}\text { Existe fácil acceso a las bases de datos para buscar investigaciones } \\
\text { Me interesa leer lo relacionado con la investigación en educación }\end{array}$ & 42.3 & 53.8 \\
$\begin{array}{l}\text { Suelo leer sobre las investigaciones educativas que se difunden en los } \\
\text { medios de comunicación }\end{array}$ & 50.0 & 38.5 \\
\end{tabular}


UT. Revista de Ciències de l'Educació

2015 num. 1. Pag. 8-29
Actitud del alumnado hacia la investigación en educación: Trabajando con vídeos en estudios de grado

Leo libros y artículos sobre investigaciones interesantes

11.5

26.9

Busco materiales audiovisuales para hacer mis investigaciones

50.0

53.8

Tabla 3. Comparativa de ambos ATR-ED en la dimensión "Medios de investigación" en los niveles de acuerdo y totalmente de acuerdo

La mayor parte del alumnado piensa que las fuentes de información sobre investigación en educación son útiles. Aumenta notablemente el porcentaje de alumnado de acuerdo y totalmente de acuerdo en los ítems "Las fuentes de información sobre investigaciones en educación son útiles" $\mathrm{y}$ "Los recursos audiovisuales fomentan el interés por la investigación educativa" en el ATR-ED posterior. Por lo tanto, el alumnado apoya el uso de recursos audiovisuales como los vídeos para aprender sobre investigación en educación. Esto indica que el vídeo es una buena herramienta para mejorar el interés del alumnado hacia un tema específico (Chen \& Cowie, 2014; Sever et al, 2013). Atendiendo al porcentaje de los demás ítems mostrados en la Tabla 3 se observa que ha aumentado el porcentaje de alumnos en los niveles citados anteriormente a excepción del ítem "Me interesa leer lo relacionado con la investigación en educación". El alumnado ha mejorado su interés hacia la investigación en educación gracias al programa seguido en la asignatura mediante vídeos (Chen et al, 2012; Hussein \& Ara, 2013; Peng et al, 2006; Sever et al, 2013; Schare et al, 1991).

\section{Conclusiones, Limitaciones e Implicaciones del Estudio}

Se puede afirmar que la actitud del alumnado hacia la investigación en educación ha mejorado. La hipótesis de partida del estudio es que los estudiantes tienen bajo interés por la investigación educativa y que los materiales audiovisuales y los canales multimedia universitarios pueden ser un excelente vehículo de propagación de información sobre investigación en educación para ayudarles a mejorar su actitud. Se acepta la hipótesis en la medida en que los resultados mostrados en el primer ATR-ED son bajos y los vídeos ayudan a mejorar la actitud de un alto porcentaje de alumnado. De acuerdo con Papanastasiou (2005), Cosgun (2013), Hussain \& Ara (2013); Bibi, Muhammad \& Majid (2013) la actitud del alumno frente a la investigación en educación se muestra baja antes de aplicar el programa de la asignatura y mejora tras la aplicación. Además, los vídeos sobre investigación en educación han ayudado a mejorar la actitud del alumnado captando su interés en el tema (Bartolomé, 2008; Blomberg et al, 2014; Chen \& Cowie, 2014; García-Fernández, 2011; Sever et al, 2013; So, Hung \& Yip, 2008).

Se pueden extraer las siguientes conclusiones de las comparativas realizadas. Por un lado, existen varias dimensiones en las que aumenta el porcentaje de los alumnos en los niveles totalmente en desacuerdo y totalmente de acuerdo, excepto en la dimensión de "Medios de investigación" que aumenta el porcentaje en el nivel de acuerdo. Esta dimensión es clave en este estudio ya que se refiere al uso de vídeos para la mejora de la actitud del alumnado frente a la investigación en educación. Por otro lado, el nivel medio ni de acuerdo ni en desacuerdo presenta mínimas diferencias entre el ATR-ED previo y el ATR-ED posterior. Estos datos significan que no todo el alumnado mejora 
su actitud. Mientras que hay un porcentaje que la mejora otro empeora su actitud. Se puede afirmar que el alumnado ha aumentado su conocimiento hacia investigación en educación y esto les lleva a modificar su actitud frente a la misma. El conocimiento en este tema ha llevado a un porcentaje de alumnado a interesarse por la investigación y a otros a desinteresarse. Aunque las actitudes positivas hacia la investigación hayan disminuido en el ATR-ED posterior (Figura 4), la dimensión "Medios de investigación" (Figura 8) ha mejorado en el ATR-ED posterior. El análisis realizado (Tabla 3) permite afirmar que el alumnado prefiere ver vídeos en vez de trabajar artículos de investigación en educación (80,8\%). Además, los vídeos activan su interés hacia este tema por lo que se puede afirmar que son herramientas de utilidad para captar la atención e interés del alumnado universitario hacia un tema, en este caso, hacia la investigación en educación. Somos conscientes de que videoartículos, redes sociales, blogs, apps en móviles y tabletas... tienen un futuro importante en la divulgación de la investigación (Vázquez-Cano, 2013).

\section{Limitaciones de la investigación y sugerencias para futuros estudios}

El estudio presenta varias limitaciones. La primera se refiere tanto al reducido número de participantes (26 estudiantes) como al muestreo realizado; probablemente un número más elevado de participantes podría permitir extrapolar los resultados a la población universitaria, también de otros grados. Se ha detectado otra limitación en el transcurso de la investigación, debido al nivel de inglés de los participantes y los vídeos recopilados sobre investigación en educación en esta universidad. Ambas limitaciones van unidas. La producción de vídeoartículos sobre investigación en educación es escasa casi nula, por lo que se ha trabajado en la asignatura con algunos vídeos en inglés. El empleo de recursos audiovisuales en inglés no ha sido acogido positivamente por el alumnado.

El estudio genera la posibilidad de continuar trabajando en varios aspectos. Sería interesante por un lado comprobar el efecto de diferentes metodologías de uso de vídeos en la difusión y visibilidad de la investigación en educación y, sobre todo, su influencia en el aprendizaje de los estudiantes de grado. Por otro lado, continuar investigando en profundidad en el cambio de actitud del alumnado y proporcionar explicaciones adicionales sobre la influencia de metodología-medio, es decir, si este cambio se debe al método "Flipped Classroom" seguido en clase (Díaz y Gallego, 2015), o bien al medio empleado, en este caso el vídeo. Ambas sugerencias permiten reflexionar sobre el tema de las actitudes del alumnado hacia la investigación desde un enfoque metodológico o desde un enfoque basado en recursos audiovisuales. Ambos sostienen la posibilidad de la creación de un canal multimedia de investigación en educación con producciones de la Universidad de Granada, lo que podría mejorar la visibilidad de la investigación en educación. Por lo tanto, el trabajo investigador del docente sería más visible para la comunidad universitaria. Además, la integración de nuevos recursos como los vídeoartículos implicaría valorar qué metodología es la más 
adecuada en este caso para favorecer el aprendizaje del alumno hacia la investigación y despertar su interés.

En resumen, se plantea una línea de investigación que integre un nuevo concepto del vídeo como son los videoartículos y una nueva metodología adaptada a este recurso.

\section{Nota}

a https://www.youtube.com/user/NoticiasCienciaUGR/videos

\section{Agradecimientos}

Este estudio ha sido subvencionado por Plan Propio de Investigación de la Universidad de Granada. Año 2014.

\section{Referencias Bibliográficas}

Ato, M., \& Vallejo, G. (2015). Diseños experimentales en Psicología. Madrid: Pirámide.

Bartolomé, A. (2008). Vídeo digital y educación. Madrid: Síntesis.

Bate, F. (2010). A bridge too far? Explaining beginning teachers' use of ICT in Australian schools. Australasian Journal of Educational Technology, 26(7), 1042-1061.

Bibi, F., Muhammad, H., \& Majid, N. (2013). Attitude of prospective teachers towards research: implications for teacher education in Pakistan. Contemporary Educational Researches Journal, 2(1), 8-14.

Bisquerra Alzina, R. (2004). Metodología de la investigación educativa. Madrid: La Muralla.

Blomberg, G., Sherin, M. G., Renkl, A., Glogger, I., \& Seidel, T. (2014). Understanding video as a tool for teacher education: investigating instructional strategies to promote reflection. Instructional Science, 42(3), 443-463. doi: 10.1007/s11251-013-9281-6

Boscolo, P. (2009). Engaging and motivating children to write. The Sage Handbook of Writing Development, 300-312. doi:10.4135/9780857021069.n21

Campbell, D. T., \& Stanley, J. C. (1973). Diseños experimentales y cuasiexperimentales en la investigación social. Buenos Aires: Amorrortu.

Cebrián, M. y Gallego, M.J. (Coords). (2011). Procesos educativos con TIC en la sociedad del conocimiento. Madrid: Pirámide. 
Chen, H. H.-J., Chen, M.-P., Chen, N.-S., \& Yang, C. (2012). Pre-Service Teachers' Views on Using Adventure Video Games for Language Learning. Proceedings of the 6th European Conference on Games Based Learning, 125-130.

Chen, J., \& Cowie, B. (2014). Scientists talking to students through videos. International Journal of Science and Mathematics Education, 12(2), 445-465. doi: 10.1007/s10763013-9415-y

Coşgun, M. (2013). Prospective teachers' engagement in educational research. Educational Research and Reviews, 8(11), 758.

Díaz-Martín, C., \& Gallego-Arrufat, M.J. (2015). Uso de videos en un innovador modelo de aprendizaje en estudios de grado. Comunicación presentada al Congreso Virtual Iberoamericano sobre Recursos Educativos Innovadores.

Duart, J.M., \& Mengual-Andrés, S. (2014). Impacto de la Sociedad del Conocimiento en la universidad y en la comunicación científica. RELIEVE-Revista Electrónica de Investigación y Evaluación Educativa, 20(2). doi: 10.7203/relieve.20.2.4343.

Ekiz, D. (2006). Primary School Teachers' Attitudes towards Educational Research. Educational Sciences: Theroy \& Practice, 6(2), 373-402.

García-Fernández, P. (2011). Aprendizaje Autónomo Utilizando Vídeos Docentes. IEEERITA, 6(2), 65-69.

Field, K. (2011). The importance of research for education's future. Recuperado de http://www.expressandstar.com/education/2011/06/14/the-importance-of-researchoreducations-future/

Gitlin, A., Barlow, L., Burbank, M.D., Kauchak, D., \& Stevens, T. (1999). Pre-service teachers' thinking on research: Implications for inquiry oriented teacher education. Teaching and Teacher Education, 15(7), 753-769.

Hussain, I., \& Ara, J. (2013). Master in Education Student Attitudes towards Research: A Comparison between two Public Sector Universities in Punjab. South Asian Studies, 28(1), 97-105.

Ibañez, P. (2013). Satisfacción y necesidades formativas de estudiantes universitarios para la elaboración y uso de mapas conceptuales. Trabajo Fin de Máster. Granada: Universidad de Granada.

Jaffar, A.A. (2012). YouTube: An emerging tool in anatomy education. Anatomical Sciences Education, 5(3), 158-164. doi: 10.1002/ase.1268

Joram, E. (2007). Clashing epistemologies: Aspiring teachers', practicing teachers', and professors' beliefs about knowledge and research in education. Teaching and Teacher Education, 23(2), 123-135. doi: 10.1016/j.tate.2006.04.032

Mora, J.G. (2004). La necesidad del cambio educativo para la sociedad del conocimiento. Revista Iberoamericana de Educación, 35, 13-37. 
Papanastasiou, E.C. (2005). Factor structure of the attitudes towards research scale. Statistics Education Research Journal, 4(1), 16-26.

Peng, H., Tsai, C. C., \& Wu, Y. T. (2006). University students' self-efficacy and their attitudes towards the Internet: the role of students' perceptions of the Internet. Educational Studies, 32(1), 73-86.

Schare, B. L., Dunn, S. C., Clark, H. M., Soled, S. W., \& Gilman, B. R. (1991). The effects of interactive video on cognitive achievement and attitude towards learning. The Journal of Nursing Education, 30(3), 109-113.

Sever, S., Oguz-Unver, A., \& Yurumezoglu, K. (2013). The effective presentation of inquiry-based classroom experiments using teaching strategies that employ video and demonstration methods. Australasian Journal of Educational Technology, 29(3), 450463.

So, W. W. M., Hung, V. H. K., \& Yip, W. Y. W. (2008). The digital video database: A virtual learning community for teacher education. Australasian Journal of Educational Technology, 24(1), 73-90.

Van der Linden, W., Bakx, A., Ros, A., Beijaard, D., \& Vermeulen, M. (2012). Student teachers' development of a positive attitude towards research and research knowledge and skills. European Journal of Teacher Education, 35(4), 401-419.

Van der Linden, W., Bakx, A., Ros, A., Beijaard, D., \& Van den Bergh, L. (2015). The development of student teachers' research knowledge, beliefs and attitude. Journal of Education for Teaching, 41(1), 4-18.

Vázquez-Cano, E. (2013). El videoartículo: nuevo formato de divulgación en revistas científicas y su integración en MOOCs. Comunicar, 41, 83-91. doi: 10.3916/C41-201308 